\title{
THE LENGTH OF THE CONTINUED FRACTION EXPANSION FOR A CLASS OF RATIONAL FUNCTIONS IN $\mathbb{F}_{q}(X)$
}

\author{
by ARNOLD KNOPFMACHER
}

(Received 6th December 1988)

A study is made of the length $L(h, k)$ of the continued fraction algorithm for $h / k$ where $h$ and $k$ are co-prime polynomials in $\mathbb{F}_{q}[X], \mathbb{F}_{q}$ a finite field. In addition we investigate the sum of the degrees of the partial quotients in this expansion for $h / k, h, k$ in $\mathbb{F}_{q}[X]$. The above continued fraction is determined by means of the Euclidean algorithm for the polynomials $h, k$ in $\mathbb{F}_{q}[X]$.

1980 Mathematics subject classification (1985 Revision): 12C05

\section{Introduction}

Let $\mathbb{F}_{q}[X]$, denote a polynomial ring in an indeterminate $X$ over a finite field $\mathbb{F}_{q}$ with exactly $q$ elements, and let $\partial=\operatorname{deg}$ denote the degree function on $\mathbb{F}_{q}[X]-\{0\}$.

It is known that, with uniqueness up to non-zero (scalar) factors in $\mathbb{F}_{q}$, the g.c.d. $\langle h, k\rangle$ of two non-zero polynomials $h, k \in \mathbb{F}_{q}[X]$ is obtainable by the Euclidean algorithm:

$$
\begin{array}{cc}
h=a_{0} k+k_{1} & \left(0 \leqq \partial k_{1}<\partial k\right), \\
k=a_{1} k_{1}+k_{2} & \left(0 \leqq \partial k_{2}<\partial k_{1}, \partial a_{1}=\partial k-\partial k_{1} \geqq 1\right), \\
\cdots & \cdots \\
k_{i-1}=a_{i} k_{i}+k_{i+1}\left(0 \leqq \partial k_{i+1}<\partial k_{i}, \partial a_{i}=\partial k_{i-1}-\partial k_{i} \geqq 1\right), \\
\quad \ldots & \cdots \\
k_{N-1}=a_{N} k_{N}+0 & \left(0 \leqq \partial k_{N}<\partial k_{N-1}, \partial a_{N}=\partial k_{N-1}-\partial k_{N} \geqq 1\right) .
\end{array}
$$

Then

$$
\langle h, k\rangle=\left\langle k_{1}, k\right\rangle=\cdots=\left\langle k_{N-1}, k_{N}\right\rangle=k_{N},
$$

and we denote the length $N$ of the algorithm by $L(h, k)$. The equivalent representation of $h / k$ as a finite continued fraction in the field of fractions $\mathbb{F}_{q}(X)$ yields an alternative interpretation of $N=L(h, k)$ as the length of the expansion 


$$
\frac{h}{k}=a_{0}+\frac{1}{a_{1}+\frac{1}{a_{2}+\cdot \cot \frac{1}{a_{N}}}}\left(\partial a_{i} \geqq 1 \text { for } i \geqq 1\right)
$$

The equations (1) also show that

$$
\partial k=\partial a_{1}+\cdots+\partial a_{N}+\partial k_{N}
$$

and we deduce that $1 \leqq L(h, k)=N \leqq \partial k$ in all cases. In [4] we investigated the average length of the Euclidean algorithm over all polynomials $h, k \in \mathbb{F}_{q}[X]$, satisfying $0 \leqq \partial h<$ $\partial k=t$. In order to state these results we need the following notation.

Definition 1. Let $E_{N}(t)=\#\left\{(h, k): L(h, k)=N, h, k \in \mathbb{F}_{q}[X]\right.$ and $\left.0 \leqq \partial h<\partial k=t\right\}, 1 \leqq$ $N \leqq t$.

Furthermore let $E(t)=\#\left\{(h, k): h, k \in \mathbb{F}_{q}[X], 0 \leqq \partial h<\partial k=t\right\}$. Also, we set $L(0, k)=0$ and

$$
E_{0}(t)=\#\left\{(h, k): h, k \in \mathbb{F}_{q}[X], h=0, \partial k=t\right\}=(q-1) q^{t} .
$$

Then, from the results in [4] the distribution of $L(h, k)$ follows a binomial law:

$$
\operatorname{Prob}\{L(h, k)=N\}=\frac{E_{N}(t)}{E(t)}=\left(\begin{array}{c}
t \\
N
\end{array}\right)\left(\frac{q-1}{q}\right)^{N}\left(\frac{1}{q}\right)^{t-N}, \quad 0 \leqq N \leqq t,
$$

with mean value $((q-1) / q) t$ and variance $\left((q-1) / q^{2}\right) t$. Kilian [3] (using a deep result of Dixon [1]), obtained asymptotic formulas for the average length of the ordinary Euclidean algorithm for integers. Equation (4) can therefore be regarded as a stronger counterpart in $\mathbb{F}_{q}[X]$ of these results. However, in the case of natural numbers, much of the literature dealing with the length of continued fractions considers only co-prime pairs of integers. See in particular Heilbronn [2] and Porter [6]. We settle now the similar problem in $\mathbb{F}_{q}[X]$.

Definition 2. Let $\mathscr{E}(t)=\left\{(h, k): h, k \in \mathbb{F}_{q}[X],\langle h, k\rangle \in \mathscr{F}_{q} \backslash\{0\}, 0 \leqq \partial h<\partial k=t\right\}$. Furthermore let

$$
\widehat{E}_{N}(t)=\#\{(h, k): L(h, k)=N,(h, k) \in \mathscr{E}(t)\}, \quad 1 \leqq N \leqq t
$$

and

$$
\hat{E}(t)=\sum_{N=1}^{t} \hat{E}_{N}(t)=\#\{(h, k): h, k \in \mathscr{E}(t)\}
$$

Theorem 3. For $t \geqq 1,1 \leqq N \leqq t$ 


$$
\hat{E}_{N}(t)=\left(\begin{array}{c}
t-1 \\
N-1
\end{array}\right)(q-1)^{N+1} q^{t}
$$

Furthermore for $1 \leqq N \leqq t$,

$$
\operatorname{Prob}\{L(h, k)=N,(h, k) \in \mathscr{E}(t)\}=\frac{\hat{E}_{N}(t)}{\hat{E}(t)}=\left(\begin{array}{c}
t-1 \\
N-1
\end{array}\right)\left(\frac{q-1}{q}\right)^{N-1}\left(\frac{1}{q}\right)^{t-1-(N-1)}
$$

Thus the distribution of $L(h, k)$ in $\mathscr{E}(t)$ follows a shifted binomial law with mean value

$$
\frac{q-1}{t} t+\frac{1}{q} \text { and variance } \frac{q-1}{q^{2}}(t-1)
$$

We observe from (4) and (6) that the average length of the Euclidean algorithm for co-prime pairs $(h, k)$ is greater by a constant $1 / q$ than the average for the general case. Partially related to the above, and also a problem of independent interest is the following: given a pair of non-zero polynomials $h, k \in \mathbb{F}_{q}[X]$ with

$$
\frac{h}{k}=a_{0}+\frac{1}{a_{1}+\frac{1}{a_{2}+\ddots}+\frac{1}{a_{M}}},
$$

let $D(h, k)=\sum_{i=1}^{M} \partial a_{i}$. It follows from (2), as in the case of $L(h, k)$, that $1 \leqq D(h, k) \leqq t$, whenever $0 \leqq \partial h<\partial k=t$. In Section 2 we also investigate the distribution of the quantities $D(h, k)$. By combining these results with those for $L h, k)$ we can deduce an expression for the average degree per digit of the polynomials " $a_{i}$ " in the continued fraction expansion of $h / k, 0 \leqq \partial h<\partial k=t$. This quantity is given by

$$
\frac{\sum D(h, k)}{\sum L(h, k)}=\frac{q}{q-1}-\frac{q}{(q-1)^{2} t}\left(1-\frac{1}{q^{2}}\right)=\frac{q}{q-1}+0\left(t^{-1}\right), \text { as } \quad t \rightarrow \infty,
$$

where the summations extend over $0 \leqq \partial h<\partial k=t$. Similarly, we show that the average degree per digit in the continued fraction expansion of co-prime polynomials $(h, k) \in \mathscr{E}(t)$ is

$$
\sum \sum(h, k)=\frac{q}{\sum L(h, k)}=\frac{q}{(q-1)+(1 / t)}=0\left(t^{-1}\right), \text { as } t \rightarrow \infty,
$$

where here the summations extend over all $(h, k) \in \mathscr{E}(t)$. These last results are somewhat analogous to those of Panov [5], who derived an asymptotic expression for the average 
value of the partial quotients in the ordinary continued fraction expansion of co-prime pairs of integers lying in a certain class.

In general the treatments of ordinary continued fractions deal with denominators of bounded rather than fixed magnitude, hence the problem of determining average lengths of the continued fraction for $h / k$ over all polynomials $h, k$ in $\mathbb{F}_{q}[X]$ with $0 \leqq \partial h<\partial k \leqq t$ is also of interest. Analogues of the above results for this larger class of pairs are derived in Section 3. The proof of Theorem 3 appears in Section 2.

\section{Proofs of Results}

In order to state the results concerning $D(h, k)$ we require some further notation.

Definition 4. For $1 \leqq N \leqq t$, define

$$
D_{N}(t)=\#\left\{(h, k): D(h, k)=N, h, k \in \mathbb{F}_{q}[X], 0 \leqq \partial h<\partial k=t\right\} .
$$

Also, since $D(0, k)=0$, let $D_{0}(t)=E_{0}(t)=(q-1) q^{t}$.

Note that by (2)

$$
D_{N}(t)=\#\left\{(h, k): h, k \in \mathbb{F}_{q}[X], 0 \leqq \partial h<\partial k=t, \partial\langle h, k\rangle=t-N\right\} .
$$

In particular, $D_{r}(t)=\hat{E}(t)$. Thus with this interpretation, $D_{N}(t)$ is independent of the Euclidean algorithm or continued fraction.

Theorem 5. For $t \leqq 1,1 \leqq N \leqq t$,

$$
D_{N}(t)=(q-1)^{2} q^{N+t-1} .
$$

It follows that $D(h, k)$ has a truncated geometric distribution

$$
\operatorname{Prob}\{D(h, k)=N\}=\frac{D_{N}(t)}{E(t)}=(q-1)\left(\frac{1}{q}\right)^{t+1-N}, \quad 1 \leqq N \leqq t,
$$

with mean value

$$
t-\frac{1}{q-1}\left(1-\frac{1}{q^{t}}\right)
$$

and variance

$$
\frac{q}{(q-1)^{2}}+0\left(\frac{t}{q^{t}}\right), \text { as } t \rightarrow \infty
$$


Let us denote the mean values of $L(h, k)$ in equations (4) and (6) by $\bar{L}(E, t)$ and $\bar{L}(\hat{E}, t)$, respectively, and the mean value of $D(h, k)$ in equation (11) by $\bar{D}(E, t)$. We can immediately deduce equations (7) and (8) since the respective ratios are given by $\bar{D}(E, t) /$ $\bar{L}(E, t)$ and $t / \bar{L}(\hat{E}, t)$.

Finally in order to combine our approach to Theorems 3 and 5 concerning $L(h, k)$ and $D(h, k)$ respectively, we define for $1 \leqq M \leqq t, M \leqq N \leqq t$,

$$
L D_{M, N}(t)=\#\left\{(h, k): h, k \in \mathbb{F}_{q}[X], 0 \leqq \partial h<\partial k=t, L(h, k)=M, D(h, k)=N\right\} .
$$

Proof of Theorem 5. Consider any $(h, k)$ with $L(h, k)=M, D(h, k)=N, 1 \leqq M \leqq N \leqq t$. By equations (1) and (2) there exists a unique set of $M+1$ polynomials $a_{1}, a_{2}, \ldots, a_{M}, k_{M}$ which satisfy

and

$$
\partial a_{1}+\partial a_{2}+\cdots+\partial a_{M}+\partial k_{M}=t, \quad \partial a_{i} \geqq 1, \quad 1 \leqq i \leqq M
$$

$$
\partial a_{1}+\partial a_{2}+\cdots+\partial a_{M}=N .
$$

The number of solutions of (13) is the number of solutions of

$$
x_{1}+x_{2}+\cdots+x_{M}=N-M
$$

in non-negative integers $x_{i}, 1 \leqq i \leqq M$. By a classical result in combinatorics (see e.g. Riordan [7]) this number is

$$
\left(\begin{array}{c}
M-1+N-M \\
N-M
\end{array}\right)=\left(\begin{array}{l}
N-1 \\
M-1
\end{array}\right)
$$

Now for each solution of (13) the number of $m$-tuples of polynomials $\left\{b_{1}, b_{2}, \ldots, b_{m}\right\}$ satisfying $\partial b_{i}=\partial a_{i}, 1 \leqq i \leqq M$ is

$$
(q-1) q^{\partial a_{1}}(q-1) q^{\partial a_{2}} \ldots(q-1) q^{\partial a_{M}}=(q-1)^{M} q^{N} .
$$

Corresponding to each of these we have $(q-1) q^{t-N}$ possibilities for $k_{M}$ by (12). Hence

$$
L D_{M, N}(t)=\left(\begin{array}{l}
N-1 \\
M-1
\end{array}\right)(q-1)^{M+1} q^{t}
$$

By Definition 7,

$$
D_{N}(t)=\sum_{M=1}^{N} L D_{M, N}(t)=q^{t} \sum_{M=1}^{N}\left(\begin{array}{l}
N-1 \\
M-1
\end{array}\right)(q-1)^{M+1}=(q-1)^{2} q^{t+N-1} .
$$

Since $E(t)=(q-1) q^{2 t}$, 


$$
D_{N}(t) / E(t)=(q-1)\left(\frac{1}{q}\right)^{t+1-N}, \quad 1 \leqq N \leqq t .
$$

Thus $\operatorname{Prob}\{D(h, k)=N\}$ has a truncated geometric distribution with mean

$$
\begin{aligned}
\bar{D}(E, t) & =\sum_{N=1}^{\imath} \frac{N D_{N}(t)}{E(t)}=\frac{(q-1)}{q^{1+t}} \sum_{N=1}^{t} N q^{N} \\
& =\frac{(q-1)}{q^{1+t}}\left\{\frac{q-(t+1) q^{t+1}+t q^{t+2}}{(q-1)^{2}}\right\} \\
& =t-\frac{1}{q-1}\left(1-\frac{1}{q^{t}}\right) .
\end{aligned}
$$

To determine the variance we must evaluate

$$
\sum_{N=1}^{t} \frac{N^{2} D_{N}(t)}{E(t)}-(\bar{D}(E, t))^{2}
$$

Now

$$
\begin{array}{r}
\sum_{N=1}^{t} \frac{N^{2} D_{N}(t)}{E(t)}=\frac{(q-1)}{q^{1+t}} \sum_{N=1}^{t} N^{2} q^{N}=\frac{t(t+2) q-(t+1)^{2}}{(q-1)}-\frac{2\left(t q^{2}-(t+1) q\right)}{(q-1)^{2}}+0\left(\frac{1}{q^{t}}\right) \\
\text { as } t \rightarrow \infty
\end{array}
$$

Next,

$$
(\bar{D}(E, t))^{2}=t^{2}-\frac{2 t}{(q-1)}+\frac{1}{(q-1)^{2}}+0\left(\frac{t}{q^{t}}\right), \quad \text { as } \quad t \rightarrow \infty
$$

By subtracting these expressions we obtain as $t \rightarrow \infty$ the required asymptotic estimate for the variance.

Proof of Theorem 3. By (2) and (8)

$$
\hat{E}(t)=D_{t}(t)=(q-1)^{2} q^{2 t-1} .
$$

The conditions $\langle h, k\rangle \in \mathbb{F}_{q} \backslash\{0\}, L(h, k)=N$ correspond to the conditions $L(h, k)=N$, $D(h, k)=t$. Thus by (14) we obtain 


$$
\hat{E}_{N}(t)=L D_{N, t}(t)=\left(\begin{array}{c}
t-1 \\
N-1
\end{array}\right)(q-1)^{N+1} q^{t}
$$

Hence

$$
\begin{aligned}
\operatorname{Prob}\{L(h, k)=N, h, k \in \mathscr{E}(t)\} & =\frac{\hat{E}_{N}(t)}{\hat{E}(t)}=\left(\begin{array}{c}
t-1 \\
N-1
\end{array}\right)(q-1)^{N-1} q^{1-t} \\
& =\left(\begin{array}{c}
t-1 \\
N-1
\end{array}\right)\left(\frac{q-1}{q}\right)^{(N-1)}\left(\frac{1}{q}\right)^{(t-1)-(N-1)}
\end{aligned}
$$

This is a binomial distribution in terms of $N-1$ for $0 \leqq N-1 \leqq t-1$. It follows that the mean value for $L(h, k)$ is $((q-1) / q)(t-1)+1$ and the variance is $\left((q-1) / q^{2}\right)(t-1)$.

\section{Averages over a larger class of polynomials}

We require some further notation, in order to state the results concerning pairs of polynomials $(h, k)$ for which $0 \leqq \partial h<\partial k \leqq t$.

Definition 6. Let $M(t)=\#\left\{(h, k): h, k \in \mathbb{F}_{q}[X], 0 \leqq \partial h<\partial k \leqq t\right\}$. Furthermore let for $0 \leqq N \leqq t$,

$$
L_{N}(t)=\#\{(h, k): L(h, k)=N, 0 \leqq \partial h<\partial k \leqq t\}
$$

and

$$
D_{N}^{*}(t)=\#\{(h, k): D(h, k)=N, 0 \leqq \partial h<\partial k \leqq t\}
$$

In [4] we derived an expression for $L_{N}(t)$ and determined the mean value over all pairs $(h, k), 0 \leqq \partial h<\partial k \leqq t$, and $h \neq 0$, to be

$$
\frac{q-1}{q}-\frac{1}{q(q+1)}+0\left(\frac{t}{q^{2}}\right), \quad \text { as } \quad t \rightarrow \infty
$$

For our purposes we prefer to include the cases for which $h=0$. A simple modification of the proof in [4] gives the mean value over all pairs $(h, k)$ with $0 \leqq \partial h<\partial k=t$ as

$$
\bar{L}(M, t)=\frac{q-1}{q} t-\frac{1}{q(q+1)}+0\left(\frac{t}{q^{2 t}}\right), \quad \text { as } \quad t \rightarrow \infty .
$$

We derive below the corresponding result in the case of co-prime pairs $(h, k)$.

Definition 7. Let $\mathscr{M}(t)=\left\{(h, k): h, k \in \mathbb{F}_{q}[X],\langle h, k\rangle \in \mathbb{F}_{q} \backslash\{0\}, 0 \leqq \partial h<\partial k \leqq t\right\}$. Furthermore let 


$$
\widehat{L}_{N}(t)=\#\{(h, k): L(h, k)=N,(h, k) \in \mathscr{M}(t)\}, \quad 1 \leqq N \leqq t
$$

and

$$
\hat{M}(t)=\#\{(h, k): h, k \in \mathscr{M}(t)\}
$$

Theorem 8. For $t \geqq 1,1 \leqq N \leqq t$,

$$
\begin{gathered}
\hat{L}_{N}(t)=(q-1)^{N+1} \sum_{r=n}^{t}\left(\begin{array}{c}
r-1 \\
N-1
\end{array}\right) q^{r} \\
=(q-1)\left\{\frac{q^{N+t}}{(N-1) !} \sum_{i=0}^{N-1} c(N-1, i) q^{-i}+(-1)^{N} q^{N}\right\},
\end{gathered}
$$

where

$$
c(N-1, i)=(-1)^{i}\left(\begin{array}{c}
N-1 \\
i
\end{array}\right) \prod_{\substack{r=0 \\
r \neq i}}^{N-1}(t-r)
$$

Further the average length of the continued fraction summing over all pairs $(h, k) \in \mathscr{M}(t)$ is

$$
\bar{L}(\hat{M}, t)=\frac{\sum L(h, k)}{\hat{M}(t)}=\frac{(q-1)}{q} t+\frac{1}{q+1}+0\left(\frac{t}{q^{2 t}}\right), \quad \text { as } \quad t \rightarrow \infty
$$

Note that for any fixed value of $N$, equation (18) is far better suited for the exact computation of $\hat{L}_{N}(t)$, than equation (17) when $t$ becomes large.

We consider next the distribution of $D_{N}^{*}(t)$.

Theorem 9. For $t \geqq 1$,

$$
D_{N}^{*}(t)= \begin{cases}(q-1) q^{N}\left(q^{t}-q^{N-1}\right), & 1 \leqq N \leqq t \\ q\left(q^{t}-1\right), & N=0\end{cases}
$$

Furthermore the mean value of $D(h, k)$ summing over all pairs $(h, k)$ with $0 \leqq \partial h<\partial k \leqq t$ is

$$
\bar{D}(M, t)=\frac{\sum D(h, k)}{M(t)}=t-\frac{q+2}{q^{2}-1}+0\left(q^{-t}\right), \quad \text { as } \quad t \rightarrow \infty
$$

In addition, the mean value of $D(h, k)$ summing over pairs $(h, k)$ in $\mathscr{M}(t)$ is

$$
\bar{D}(\hat{M}, t)=\frac{\sum D(h, k)}{\widehat{M}(t)}=t-\frac{1}{q^{2}-1}+0\left(\frac{t}{q^{2 t}}\right), \quad t \rightarrow \infty
$$


Using the above results, the average degree per digit in the continued fraction expansion of $h / k$ for co-prime pairs $(h, k) \in \mathscr{M}(t)$ is

$$
\frac{\bar{D}(\hat{M}, t)}{\bar{L}(\hat{M}, t)}=\frac{q}{q-1}\left(1-\frac{1}{t(q-1)}\right)+0\left(t^{-2}\right), \quad \text { as } \quad t \rightarrow \infty .
$$

Similarly, the average degree per digit in the continued fraction expansion of $h / k$ over all pairs with $0 \leqq \partial h<\partial k \leqq t$ is

$$
\frac{\bar{D}(M, t)}{\bar{L}(M, t)}=\frac{q}{q-1}\left(1-\frac{1}{t(q-1)}\right)+0\left(t^{-2}\right), \quad \text { as } \quad t \rightarrow \infty .
$$

Proof of Theorem 8. By definition of $\hat{L}_{N}(t)$ and $\hat{E}_{N}(t)$,

$$
\hat{L}_{N}(t)=\sum_{r=1}^{t} \hat{E}_{N}(r)
$$

Hence by Theorem 3

$$
\hat{L}_{N}(t)=(q-1)^{N+1} \sum_{r=N}^{t}\left(\begin{array}{c}
r-1 \\
N-1
\end{array}\right) q^{r}
$$

Now using the result (see [4]),

$$
(q-1)^{N+1} \sum_{r=N}^{t}\left(\begin{array}{c}
r \\
N
\end{array}\right) q^{r}=\frac{q^{N+i+1}}{N !} \sum_{i=0}^{N} c(N, i) q^{-i}+(-1)^{N-1} q^{N}
$$

where

$$
c(N, i)=(-1)^{i}\left(\begin{array}{c}
N \\
i
\end{array}\right) \sum_{\substack{r=0 \\
r \neq i}}^{N}(t+1-r)
$$

the other formula for $\hat{L}_{N}(t)$ follows.

To determine the average value it is simplest to use $\bar{L}(\hat{E}, t)$. By definition,

$$
\bar{L}(\hat{M}, t)=\frac{\sum_{(h, k) \in \mathcal{M}(t)} L(h, k)}{\hat{M}(t)} .
$$

Now

$$
\sum_{(h, k) \in \mathcal{M}(t)} L(h, k)=\sum_{r=1}^{t} \sum_{\substack{(h, k) \in \mathcal{S}(t) \\ \partial k=r}} L(h, k)=\sum_{r=1}^{t} \bar{L}(\hat{E}, r) \hat{E}(r)
$$


16

A. KNOPFMACHER

$$
\left.=\frac{(q-1)}{(q+1)^{2}}\left\{1-(t+1) q^{2 t}+t q^{2 t+2}\right\}+\frac{(q-1)}{(q+1)}\left(q^{2 t}-1\right) \quad \text { (by Theorem } 3\right) .
$$

Next,

$$
\hat{M}(t)=\sum_{r=1}^{t} \hat{E}(r)=\frac{(q-1)^{2} q\left(q^{2 t}-1\right)}{q^{2}-1} .
$$

Combining these results,

$$
\begin{aligned}
\vec{L}(\hat{M}, t) & =\frac{1}{\left(1-q^{-2 t}\right)}\left(\frac{q-1}{q} t-\frac{1}{q(q+1)}\right)+\frac{1}{q}\left(1+\frac{1}{(q+1)\left(q^{2 t}-1\right)}\right) \\
& =\frac{q-1}{q} t+\frac{1}{q+1}+0\left(\frac{t}{q^{2 t}}\right), \quad \text { as } t \rightarrow \infty .
\end{aligned}
$$

Proof of Theorem 9. By definition, for $1 \leqq N \leqq t$,

$$
D_{N}^{*}(t)=\sum_{r=N}^{t} D_{N}(r)=(q-1)\left(q^{N+t}-q^{2 N-1}\right)
$$

Similarly, $D_{0}^{*}(t)=q\left(q^{t}-1\right)$. Next

$$
M(t)=\sum_{r=1}^{t} E(r)=\frac{(q-1) q^{2}\left(q^{2 t}-1\right)}{q^{2}-1} .
$$

Thus

$$
\begin{aligned}
\bar{D}(M, t) & =\sum_{N=1}^{t} N D_{N}^{*}(t) / M(t)=\frac{(q-1)}{M(t)}\left\{q^{t} \sum_{N=1}^{t} N q^{N}-\sum_{N=1}^{t} N q^{2 N-1}\right\} \\
& =\frac{1}{\left(1-q^{-2 t}\right)}\left(t-\frac{(q+2)}{\left(q^{2}-1\right)}\right)+\frac{1}{\left(q^{2 t}-1\right)}\left(q^{t-1}\left(q^{2}-1\right)-\frac{1}{q\left(q^{2}-1\right)}\right) \\
& =t-\frac{q+2}{q^{2}-1}+O\left(q^{-t}\right), \quad \text { as } t \rightarrow \infty .
\end{aligned}
$$

Finally,

$$
\bar{D}(\hat{M}, t)=\sum_{r=1}^{t} r \hat{E}(r) / \hat{M}(t)=\frac{t}{1-q^{-2 t}}-\frac{1}{q^{2}-1}
$$

https://doi.org/10.1017/S001309150000496X Published online by Cambridge University Press 


$$
=t-\frac{1}{q^{2}-1}+0\left(\frac{t}{q^{2 t}}\right), \quad \text { as } \quad t \rightarrow \infty .
$$

In addition to the mean values, formulas (17) and (20) can in principle be used to determine precisely the variances of $L(h, k)$ and $D(h, k)$, for the respective classes of pairs $(h, k)$.

Acknowledgement. I would like to thank J. L. Nicolas for demonstrating to me that the results of [4] could be derived more simply by a combinatorial approach. I have used this approach in proving these further results.

\section{REFERENCES}

1. J. D. Dixon, The numbers of steps in the Euclidean algorithm, J. Number Theory 2 (1970), 414-422.

2. H. Heilbronn, On the average length of a class of finite continued fractions, in Number Theory and Analysis (Plenum Press, New York, 1969), 87-96.

3. H. Killian, Zur mittleren Anzahl von Schritten beim eukklidischen Algorithmus, Elem. Math. 38 (1983), 11-15.

4. A. KNOPFmaCher and J. KNOPFMacher, The exact length of the Euclidean algorithm in $\mathbb{F}_{q}[X]$, Mathematika 35 (1988), 297-304.

5. A. A. Panov, Averages over elements of a certain class of finite continued fractions, Russian Math. Surveys 35 (1980), 182-183.

6. J. W. Porter, On a theorem of Heilbronn, Mathematika 22 (1975), 20-28.

7. J. Riordan, An Introduction to Combinatorial Analysis (Princeton University Press, Princeton, N.J., 1978).

Department of Computational and Applied Mathematics

UNIVERSITY OF THE WITWATERSRAND

JoHANNESBURG, P O WiTs 2050

South Africa 